\title{
Consequences of a strong phase transition in the dense matter equation of state for the rotational evolution of neutron stars
}

\author{
M. Bejger ${ }^{1}$, D. Blaschke ${ }^{2,3,4}$, P. Haensel ${ }^{1}$, J. L. Zdunik ${ }^{1}$, and M. Fortin ${ }^{1}$ \\ ${ }^{1}$ Nicolaus Copernicus Astronomical Center, Polish Academy of Sciences, Bartycka 18, 00-716 Warsaw, Poland \\ e-mail: [bejger; haensel; jlz; fortin]@camk.edu.pl \\ 2 Institute of Theoretical Physics, University of Wroclaw, pl. Maxa Borna 9, 50-204 Wroclaw, Poland \\ 3 Bogoliubov Laboratory for Theoretical Physics, JINR Dubna, ul. Joliot-Curie 6, 141980 Dubna, Russia \\ ${ }^{4}$ National Research Nuclear University (MEPhI), Kashirskoe Shosse 31, 115409 Moscow, Russia \\ e-mail: blaschke@ift.uni.wroc.pl
}

Received 24 August 2016 / Accepted 9 December 2016

\begin{abstract}
Aims. We explore the implications of a strong first-order phase transition region in the dense matter equation of state in the interiors of rotating neutron stars, and the resulting creation of two disjoint families of neutron-star configurations (the so-called high-mass twins).

Methods. We numerically obtained rotating, axisymmetric, and stationary stellar configurations in the framework of general relativity, and studied their global parameters and stability.

Results. The instability induced by the equation of state divides stable neutron star configurations into two disjoint families: neutron stars (second family) and hybrid stars (third family), with an overlapping region in mass, the high-mass twin-star region. These two regions are divided by an instability strip. Its existence has interesting astrophysical consequences for rotating neutron stars. We note that it provides a natural explanation for the rotational frequency cutoff in the observed distribution of neutron star spins, and for the apparent lack of back-bending in pulsar timing. It also straightforwardly enables a substantial energy release in a mini-collapse to another neutron-star configuration (core quake), or to a black hole.
\end{abstract}

Key words. stars: neutron - equation of state - pulsars: general

\section{Introduction}

Recent observations of the high-mass pulsars PSR J16142230 (Demorest et al. 2010; Fonseca et al. 2016) and PSR J0348+0432 (Antoniadis et al. 2013) with masses $M \approx 2 M_{\odot}$ has motivated the nuclear and particle physics communities to deepen their understanding of the equation of state (EOS) of high-density matter and of the possible role of exotic states of matter in neutron star (NS) interiors. For further constraints on the high-density EOS from NS and heavy-ion collision experiments see Klähn et al. (2006, 2012).

Measurements of high masses of NSs do not immediately imply that very high densities prevail in their cores, meaning that a transition to exotic forms of matter (hypernuclear matter, quark matter) has to be invoked. Of two models for the highdensity EOS with the same stellar mass, the stiffer model will lead to a lower central density but to a larger radius. Therefore, radius measurements for high-mass pulsars are of the utmost importance.

Current radius measurements are controversial. Determinations of the radius $R$ range from about $9 \mathrm{~km}$ (Guillot et al. 2013) to $15 \mathrm{~km}$ (Bogdanov 2013), but one must be aware of possible systematic flaws (see, e.g., Heinke et al. 2014; Elshamouty et al. 2016); for a recent critical assessment see, for example, Fortin et al. (2015), Miller \& Lamb (2016), Haensel et al. (2016). At a gravitational mass of $\sim 2 M_{\odot}$, the range of radius values mentioned above would correspond to a range of central densities of the compact star between 2.5-6 $n_{0}$, where $n_{0}=0.15 \mathrm{fm}^{-3}$ is the nuclear saturation density.

Several observational programs for simultaneous measuring of pulsar masses and radii are currently in preparation: the Neutron star Interior Composition ExploreR (NICER, Arzoumanian et al. 2014), the Square Kilometer Array (SKA, Watts et al. 2015), Athena (Motch et al. 2013), and possibly, a LOFT-size mission (Feroci et al. 2012). Thus there is hope that in the near future it will be possible to reconstruct the cold NS matter EOS $P(\varepsilon)$ (here $P$ is the pressure and $\varepsilon$ is the energy density) within the measurement errors from the measured $M(R)$ relation by means of inverting the Tolman-Oppenheimer-Volkoff (TOV, Tolman 1939; Oppenheimer \& Volkoff 1939) equations. In addition, specific proposals to use gravitational waves (GWs) for measuring the NS radius from either the observations of the inspiral (e.g., Bejger et al. 2005; Damour et al. 2012) or postmerger waveforms (e.g., Bauswein et al. 2015) were presented.

The systematic investigation of a wide class of hybrid stars with varying stiffness of hadronic matter at high densities and the possibility of quark matter with varying high-density stiffness has revealed interesting findings (Alvarez et al. 2016). Possible future measurements of radii of recently discovered high-mass stars (Demorest et al. 2010; Antoniadis et al. 2013) would select a hybrid EOS with a strong first-order phase transition if the outcome of their radius measurement were to show a difference of about $2 \mathrm{~km}$ with significance. Such a possibility has been suggested earlier on the basis of a new class of hybrid star EOS that fulfill the generic condition that the baryonic EOS is strongly 
stiffened at high densities, for instance, by effects of the Pauli exclusion principle (quark exchange interaction between baryons), and a strong first-order deconfinement phase transition requiring sufficiently soft quark matter at the transition between baryonic and quark phases, $\mathrm{B} \longrightarrow \mathrm{Q}$. The quark matter EOS has, however, to stiffen quickly with increasing density so that immediate gravitational collapse that is due to the transition does not occur, allowing stable hybrid stars to exist.

Such a solution for hybrid stars, which form a third family of compact stars that are disconnected from the baryonic branch of compact stars, is very interesting for the possible observational verification of specific features of phase transition to quark matter in NS cores. A sharp first-order phase transition between pure $\mathrm{B}$ and Q phases, occurring at constant pressure $P_{\mathrm{BQ}}$, is associated with an energy density jump from $\varepsilon_{\mathrm{B}}$ to $\varepsilon_{\mathrm{O}}$ ( $\varepsilon$ is the energy density including the rest energy of particles). Then, a general necessary condition for the existence of a disconnected family of NSs with Q-phase cores is $\varepsilon_{\mathrm{Q}} / \varepsilon_{\mathrm{B}}>\lambda_{\text {crit }}$, with $\lambda_{\text {crit }}=\frac{3}{2}\left(1+P_{\mathrm{BQ}} / \varepsilon_{\mathrm{B}}\right)$ (Seidov 1971). The second term in the brackets comes from general relativity. The condition implies that hybrid stars with small quark cores are unstable to radial perturbations and either collapse into black holes $(\mathrm{BH})$, or to configurations located on a separate hybrid stars branch (a detailed review of the structure and stability of NSs with phase transitions in their cores is given in Sect. 7 of Haensel et al. 2007). This corresponds to a separate segment of the $M(R)$ relation. High mass and small radii imply high spacetime curvature and strong gravitational pull, resulting in a relatively flat (nearly horizontal) $M(R)$ segment. This hybrid star branch is characterized by a narrow range of $M$, a broad range of $R$, with $M$ weakly increasing with decreasing $R$, up to a very flat $M$ maximum. These features of the hybrid-star branch result in specific observational signatures of a strong $\mathrm{B} \longrightarrow \mathrm{Q}$ first-order phase transition in NS cores. Moreover, these generic properties of the hybrid-star branch indicate a relative "softness" of their configurations with respect to perturbations that are due to rotation and oscillations, for example. All these generic features are studied in the present paper, using an illustrative example of an advanced EOS composed of a stiff baryonic segment and a strong first-order phase transition to the quark phase (Sect. 3).

The $\mathrm{B} \longrightarrow \mathrm{Q}$ phase transition might occur smoothed within a finite pressure interval through a mixed BQ phase layer. However, the interplay of the surface tension at the B-Q interface and charge screening of Coulomb interaction (in a mixed state, B and $\mathrm{Q}$ phases are electrically charged) make the mixed-state layer very thin (Endo et al. 2006) so that the key features of the hybridstar branch remain intact (Alvarez-Castillo \& Blaschke 2015).

Stable branches of static configurations in the $M-R$ plane have very specific generic features (see, e.g., Benic et al. 2015). The high-mass baryon branch is very steep, not only nearly vertical, but even with $R$ increasing with $M$, a feature characteristic of very stiff NS cores. The hybrid stable twin-branch is predicted to be flat, nearly horizontal with very broad maximum. Measuring radii of $\sim 2 M_{\odot}$ stars, which span a wide range of values from about 12 to $15 \mathrm{~km}$, will clearly indicate a hybrid branch, and if moreover the radii for standard NS masses, 1.2-1.6 $M_{\odot}$, are roughly constant at approximately $14 \mathrm{~km}$, then the evidence for two distinct families that are separated as a result of a strong firstorder phase transition would be quite convincing. The range of central pressures for NSs of different radii at $2 M_{\odot}$, which may bear a connection to the universal hadronization pressure found in heavy-ion collisions, is discussed in Alvarez-Castillo et al. (2016).
This possibility of finding observational evidence for a firstorder phase transition in NS cores from the phenomenology of $M-R$ characteristics of NS populations offers the chance to answer to the currently controversial question of whether a critical endpoint of first-order phase transitions in the QCD phase diagram exists (Alvarez-Castillo \& Blaschke 2013; Blaschke et al. 2013).

Since the nature of the QCD transition at vanishing baryon density is beyond doubt identified as a crossover in two independent lattice QCD simulations at the physical point (Bazavov et al. 2014; Borsanyi et al. 2014), the evidence for a first-order phase transition at zero temperature and finite baryon density necessarily implies the existence of a critical endpoint (CEP) of first-order phase transitions in the QCD phase diagram (we note that there are theoretical conjectures about a continuity between hadronic and quark matter phases at low temperatures and finite densities in the QCD phase diagram (Schaefer \& Wilczek 1999; Hatsuda et al. 2006; Abuki et al. 2010); if, however, there were observational evidence for a horizontal branch in the $M-R$ diagram of compact stars, these considerations would become obsolete). The very existence of such a CEP is a landmark for identifying the universality class of QCD, and due to its importance for model building and phenomenology, a major target of experimental research programs with ultra-relativistic heavy-ion collisions at BNL RHIC (STAR beam energy scan, Stephans 2006) and CERN SPS (NA49future Collaboration, Gazdzicki et al. 2006), in future at NICA in Dubna (Sissakian et al. 2006) and at FAIR in Darmstadt (Friman et al. 2011).

First examples for microscopically founded hybrid star EOS that simultaneously fulfill the above constraints for the existence of a disconnected hybrid star branch and for a high gravitational mass of about $2 M_{\odot}$ (which implies the existence of socalled high-mass twin stars) have been given in Blaschke et al. (2013). The recent systematic investigation of high-mass twin stars in Alvarez-Castillo \& Blaschke (2015) is based on the EOS developed in Benic et al. (2015), which joins a relativistic density functional for nuclear matter with nucleonic excluded volume stiffening with a Nambu-Jona-Lasinio (NJL) type model for quark matter that provides a high-density stiffening as a result of higher order quark interactions. These examples belong to a new class of EOS that can be considered as a realization of the recently introduced three-window picture for dense QCD matter (Kojo 2016) as a microscopic foundation for the hybrid EOS that was conjectured by Masuda et al. (2013), suggesting a crossover construction between hadronic and quark matter. These three windows depicted in Fig. 1 of Kojo (2016) are characterized by the following:

- at densities $n_{\mathrm{b}}<2 n_{0}$ : occasional quark exchange between separated and still well-defined nucleons, leading to quark Pauli blocking effects in dense hadronic matter (Röpke et al. 1986) that can be modeled by a hadronic excluded volume or by repulsive short-range interactions;

- densities $2 n_{0}<n_{\mathrm{b}}<5 n_{0}$ : multiple quark exchanges that lead to a partial delocalization of the hadron wavefunctions and to the formation of multi-quark clusters and a softening of the EOS by an attractive mean field;

- densities $n_{\mathrm{b}}>5 n_{0}$ : baryon wave functions overlap and quarks become delocalized; a Fermi sea for strongly interacting quark matter forms with higher order repulsive short-range interactions, for example, by multipomeron exchange (Yamamoto et al. 2016) or nonlinear quark interactions (Benic 2014). 
A still open and controversial question in this context is whether chiral symmetry restoration and deconfinement transition (which both coincide on the temperature axis according to lattice QCD simulations) would also occur simultaneously in the dense matter at zero temperature. If this were not the case, then there would be room for a more complex structure of the QCD phase diagram, for instance, with a triple point that is either due to a quarkyonic matter phase (light quarks confined in baryons that form parity doublets, McLerran \& Pisarski 2007) or a massive quark matter phase (Schulz \& Röpke 1987) for which there is circumstantial evidence from particle production in ultrarelativistic heavy-ion collision experiments (Andronic et al. 2010). More circumstantial evidence for a region of strong first-order phase transitions in the QCD phase diagram comes from the baryon stopping signal in the energy dependence of the curvature of the net proton rapidity distribution at midrapidity for energies in the intermediate range between former AGS experiments and the NA49 experiment at the CERN SPS (Ivanov 2013). This signal has been proven to be quite robust under different experimental constraints (Ivanov \& Blaschke 2015) and against hadronic final state interactions (Batyuk et al. 2016).

On the basis of this discussion, the new class of EOS with a three-window structure provides the theoretical background for a strong first-order phase transition, the phenomena in the energy scan of heavy-ion collision experiments, and the creation of two disjoint families of NS configurations. Astrophysical observations of the NS twins with drastically different core compositions may be regarded as a manifestation of these dense matter EOS features in different regions of the QCD phase diagram and under different physical conditions.

In the present work we add to the discussion of high-mass twin stars a detailed investigation of their properties under rigid rotation. This is because we expect a strong response of both branches to rotation. First, the high stiffness of the high-density EOS of the baryonic branch results in large radii: $R \sim 15 \mathrm{~km}$ at $M \sim 2 M_{\odot}$, so that the effect of the centrifugal force will be large. Second, the stable static hybrid branch is flat, which makes it particularly susceptible to the effects of rotation: the margin of stability along this branch is narrow.

The neutron star instability induced by a strong phase transition in the EOS was studied in detail by Zdunik et al. (2006), who conjectured that the character of stability is not changed by the rotation rate of the star (disjoint families remain separated at any rotation rate). This question will be of particular interest for discussing compact star phenomena tied to the evolution of their rotational state, which eventually ends by collapse into a black hole (e.g., Falcke \& Rezzolla 2014).

The general idea of the present work is to illustrate generic features of a class of high-density quark phase transition EOSs using an exemplary EOS, in order to discuss the regions of stable and unstable configurations related to a strong first-order phase transition. For illustration, we use one of the EOS that was recently developed by Benic et al. (2015).

The article is structured as follows. In Sect. 2 we describe the methods we used to calculate the rotating configurations, stressing particularly the need for high precision of numerical simulations. Precision is particularly important for testing stability criteria of stationary rotating configurations, which are formulated there. Section 3 starts with a brief presentation of the EOS that we use to illustrate generic properties of the rotating high-mass twins. Then we construct families of rotating configurations, assess their stability, and classify the regions of instability and their generic features. Discussion of the results in Sect. 4 involves evolutionary considerations, potential scenarios leading to observational manifestations of massive-twin case, including dynamical phenomena triggered by the instabilities and their possible astrophysical appearances. The final part of Sect. 4 presents the conclusions.

\section{Methods}

In order to analyze the astrophysical consequences of the abovementioned specific type of EOS, we have obtained rigidly rotating, stationary, and axisymmetric NS configurations by means of the numerical library LORENE ${ }^{1}$ nrotstar code, using the $3+1$ formulation of general relativity of Bonazzola et al. (1993), and employing the multidomain pseudo-spectral decomposition (three domains inside the star). The accuracy is controlled by a 2D general-relativistic virial theorem (Bonazzola \& Gourgoulhon 1994) and for the results presented here is typically on the order of $10^{-7}$. The high accuracy provided by the spectral method implementation of LORENE and a general low numerical viscosity of spectral methods is particularly suitable for studying the stability of NS models.

The evanescent error behavior of the solutions can be employed by expanding the number of coefficients to obtain the relevant values practically up to machine precision. In this context, we recall that a global parameter that is strictly conserved during the evolution of an isolated NS is its total baryon number (baryon charge) $A_{\mathrm{b}}$. Instead of $A_{\mathrm{b}}$ it is convenient to use in relativistic astrophysics the baryon mass (or rest mass) of the NS defined by $M_{\mathrm{b}}=A_{\mathrm{b}} m_{\mathrm{b}}$, where $m_{\mathrm{b}}$ is a suitably defined mass of a single baryon. $M_{\mathrm{b}}$ is the total mass of $A_{\mathrm{b}}$ non-interacting baryons; it is easy to extend these definitions to NS cores built of quarks (three quarks contribute +1 to the baryon number of NS). In our calculations we follow the LORENE unit convention and use a value of the mean baryon mass $m_{\mathrm{b}}=1.66 \times 10^{-24} \mathrm{~g}$. Other definitions of $m_{\mathrm{b}}$, suitable for specific applications in NS and supernova physics, are discussed in Sect. 6.2 of Haensel et al. (2007). We note that while $M_{\mathrm{b}}$ is strictly constant in spinning down or cooling isolated NS, their gravitational mass is changing. We are in general interested in obtaining accurate values of the gravitational mass $M$, the baryon mass $M_{\mathrm{b}}$ and, the total angular momentum $J$ that are the properly defined functionals of the stellar structure and EOS suitable to study instabilities (for more details and a discussion comparing the $3+1$ formulation with the slow-rotation formulation see the recent review of the stability of rotating NSs with exotic cores, Haensel et al. 2016).

In the following we use the method described in Zdunik et al. (2004, 2006), who studied, among other things, the backbending phenomenon proposed for NSs in Glendenning et al. (1997). Back-bending, a temporary spin-up of an isolated NS that is decreasing its total angular momentum $J$ by the dipole radiation, for example, can be robustly quantified by analyzing the extrema of the baryon mass $M_{\mathrm{b}}$ along the lines of constant spin frequency $f$, that is, the rate of the baryon mass $M_{\mathrm{b}}$ change with respect to a central EOS parameter $\lambda_{c}$ (central pressure $P_{c}$, e.g.,) changing along $f=$ const. lines. The condition for back-bending to occur is

$$
\left.\left(\frac{\partial M_{\mathrm{b}}}{\partial \lambda_{c}}\right)\right|_{f}<0
$$

(see also Haensel et al. 2016, Sect. 7 for detailed description). Nevertheless, the susceptibility to back-bending does not mean that the NS is indeed unstable. In order to study the regions of

\footnotetext{
http: //www. lorene. obspm. fr
} 
true instability, we use the turning-point theorem formulated for rotating stellar models by Sorkin $(1981,1982)$, Friedman et al. (1988), which states that the sufficient condition for the change in stability corresponds to an extremum of the gravitational mass $M$, or the baryon mass $M_{\mathrm{b}}$ at fixed $J$ :

$$
\left.\left(\frac{\partial M}{\partial \lambda_{c}}\right)\right|_{J}=0,\left.\quad\left(\frac{\partial M_{\mathrm{b}}}{\partial \lambda_{c}}\right)\right|_{J}=0,
$$

or equivalently, to an extremum of $J$ at fixed either $M$, or $M_{\mathrm{b}}$ :

$$
\left.\left(\frac{\partial J}{\partial \lambda_{c}}\right)\right|_{M}=0,\left.\quad\left(\frac{\partial J}{\partial \lambda_{c}}\right)\right|_{M_{\mathrm{b}}}=0 .
$$

In the following, we illustrate the generic features of the third family of compact stars with one of the EOSs developed by Benic et al. (2015). The baryon phase EOS is based on the relativistic mean-field (RMF) model DD2 of Typel et al. (2010). It fits the semi-empirical parameters of nuclear matter at saturation well, and features density-dependent coupling constants. In order to obtain a high-density stiffening of B phase, which is necessary to yield a strong $\mathrm{B} \longrightarrow \mathrm{Q}$ phase transition, while fulfilling $M_{\max }>2 M_{\odot}$ for the hybrid Q branch, the DD2 model is modified using excluded volume (EV) effects that are included to account for the finite size of baryons, resulting in a DD2+EV model for the baryon phase. The EV effect was included without altering the good fit of DD2 to semi-empirical nuclear matter parameter values. The quark phase description is based on the Nambu-Jona-Lasinio density-dependent model, with dimensionless coupling constants $\eta_{2}=0.12$ and $\eta_{4}=5$. The complete EOS with a strong first-order phase transition $\mathrm{B} \longrightarrow \mathrm{Q}$ was obtained using Maxwell construction. We analyze the features of sequences of configurations along the lines of fixed baryon mass $M_{\mathrm{b}}$ and total angular momentum $J$.

\section{Results}

The static configurations of NSs for the sample EOS have been studied in Benic et al. (2015). The basic stellar parameters were the gravitational mass $M$ and the circumferential radius $R$. To analyze the stability of rotating stars, it is more convenient to consider the baryon mass $M_{\mathrm{b}}$ (also called the rest mass) of the star and the equatorial circular radius $R_{\text {eq }}$ (see, e.g., Haensel et al. 2016). Generally, the study of rapidly rotating star configurations offers advantages for the investigation of phase transitions in NS interiors because as a function of the angular momentum (rotation frequency), which is an additional parameter, the profile of the density distribution and therefore also the interior composition would change, which is expected to allow for observational signatures in the course of the rotational evolution of the star (for early examples, see Glendenning et al. 1997; Chubarian et al. 2000). The spin frequency range we studied spans the astrophysically relevant range from $f=0 \mathrm{~Hz}$ (static configurations, corresponding to the solutions of the Tolman-Oppenheimer-Volkoff equations), to $f=1000 \mathrm{~Hz}$ (i.e., much higher than the frequency of $716 \mathrm{~Hz}$ of the most rapid pulsar known to date, PSR J17482446ad of Hessels et al. 2006).

Stationary uniformly rotating configurations are labeled (determined) by two parameters. In Fig. 1 we show various continuous two-parameter curves $\left[M_{\mathrm{b}}\left(\lambda_{\mathrm{c}}, \beta\right), R_{\mathrm{eq}}\left(\lambda_{\mathrm{c}}, \beta\right)\right]$ in the $M_{\mathrm{b}}-$ $R_{\text {eq }}$ plane. Here, the parameter $\lambda_{\mathrm{c}}$ can for instance be the central pressure $P_{\mathrm{c}}$, or central baryon chemical potential $\mu_{\mathrm{b}(\mathrm{c})}$ (both behaving continuously and monotonously along the curve). The quantity $\beta$ characterizes the uniform rotation of the star; we

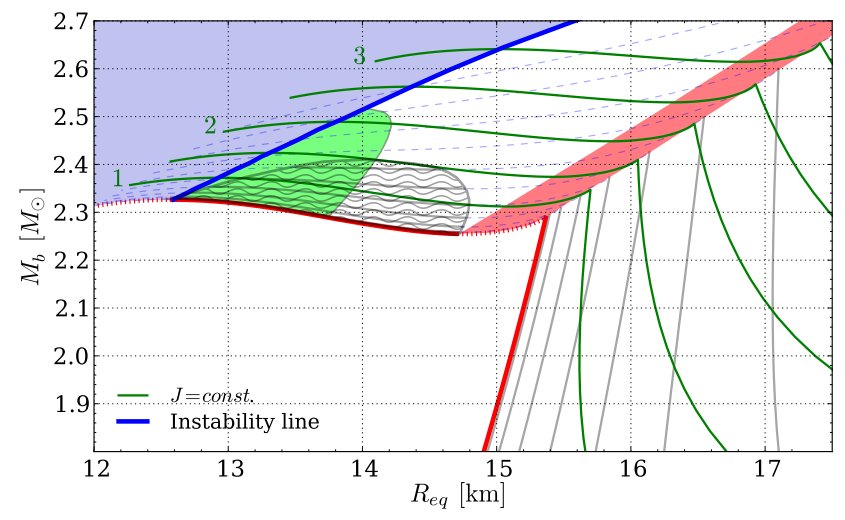

Fig. 1. Stable and unstable regions on the baryon mass-equatorial radius $M_{\mathrm{b}}-R_{\text {eq }}$ plane. The lowest (red) line denotes the nonrotating configurations. The wavy pattern region denotes a region in which the backbending phenomenon does not occur, whereas dashed lines are constant frequency tracks in a region in which back-bending occurs (lines every $100 \mathrm{~Hz}$, in the range $100-1000 \mathrm{~Hz}$ ). The solid green lines denote sequences of configurations with constant angular momentum $J$ $\left(1,1.5, \ldots, 3\right.$, in units of $\left.G M_{\odot}^{2} / c\right)$. The thick solid blue line on the left side marks the boundary of the region in which rotating axisymmetric stars are unstable with respect to axisymmetric perturbations. The red strip on the right is the instability induced by the EOS, dividing the $M_{\mathrm{b}}-R_{\mathrm{eq}}$ plane into two disjoint families of solutions. Finally, the green region is the allowed twin branch part of the $M_{\mathrm{b}}-R_{\mathrm{eq}}$ plane to where NSs collapse after entering the instability strip (assuming $M_{\mathrm{b}}=$ const. and $J=$ const.).

chose it to be equal to the frequency of rotation $f$ or the total stellar angular momentum $J$. In Fig. 1 we study the regions of backbending and stability on the baryon mass $M_{\mathrm{b}}$-equatorial radius $R_{\text {eq }}$ plane. The region in which the back-bending phenomenon is present is marked with the dashed $f=$ const. lines. An isolated NS $\left(M_{\mathrm{b}}=\right.$ const. $)$ that decreases its angular momentum (by electromagnetic dipole radiation, e.g.) crosses the lines of $f=$ const. while moving from the right to the left side of Fig. 1. According to Eq. (1), in the region of dashed lines, the $M_{\mathrm{b}}=$ const. line crosses the $f=$ const. curves such that it results in a spin-up while the angular momentum is monotonically decreasing, that is, the back-bending. The wavy pattern region denotes the opposite, usually observed behavior, that is, spin-down with angular momentum loss.

Figure 1 also shows two instability regions. The first is the familiar instability with respect to axisymmetric perturbations related to the existence of the maximum mass; the blue line corresponding to maxima of $J$ and denotes its boundary. The second instability is induced by the strong phase transition (red strip between the local minimum and maximum of $J$ ). The latter divides the space of stable solutions into two disjoint families at any rotation rate (Zdunik et al. 2006). Assuming that at some moment in its evolution an isolated NS enters the instability strip, it becomes then unstable and collapses to another, more compact configuration along the lines of $M_{\mathrm{b}}=$ const. and $J=$ const. (Dimmelmeier et al. 2009).

Configurations that survive such a mini-collapse are located in the green region. We note that in this idealized picture no mass and angular momentum loss is assumed. Realistically, some mass and angular momentum loss may occur, and so the green region will decrease toward lower spin rates and toward the region that is stable against the back-bending, which is marked with the wavy pattern in Fig. 1. Careful analysis of Fig. 1 reveals the existence of a critical angular momentum $J_{\text {crit }}$, such 

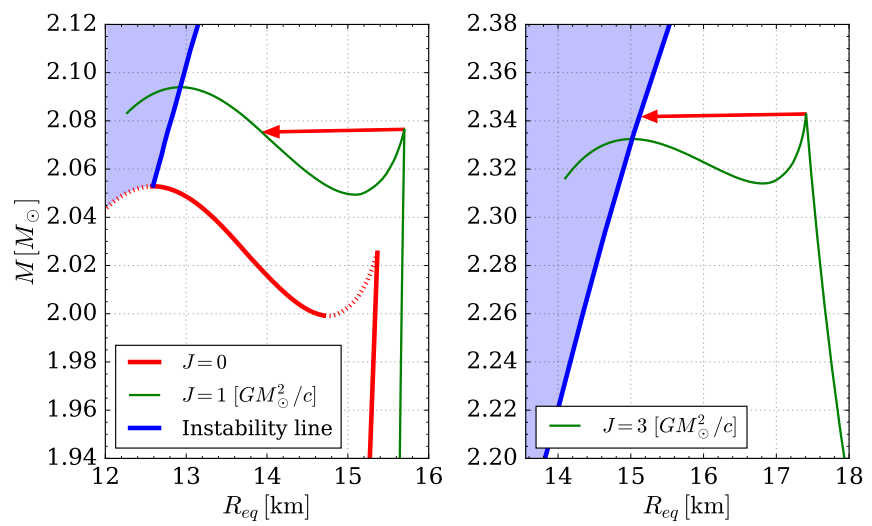

Fig. 2. Catastrophic mini-collapse for two configurations with different values of angular momentum $J$ at the edge of the instability region, on the gravitational mass-equatorial radius $M-R_{\text {eq }}$ plane. For an isolated star, the left panel shows the trajectory along a line of constant baryon mass $M_{\mathrm{b}}$ (note the arrow is inclined, since the ordinate is $M$, not $M_{\mathrm{b}}$ ) to another stable NS configuration with $J$ equal to the initial one. Right panel: if the angular momentum is larger than some critical value that depends on the EOS, the star collapses directly to a $\mathrm{BH}$, since the mass of the twin branch counterpart is too low.

that for $J>J_{\text {crit }}$ exceeding the maximum mass on the B branch (resulting from accretion) implies in a direct collapse into a rotating BH. This situation is depicted in detail in Fig. 2.

The existence of $J_{\text {crit }}$ and its potential observational signatures are discussed in more detail in Sect. 4. Here we restrict ourselves to a few comments on how the existence of $J_{\text {crit }}$ is due to generic features of the rotating-twin $M\left(R_{\mathrm{eq}}\right)$ curves. Consider first the static $(J=0) \mathrm{B}$ and Q branches. They have very different $M(R)$ dependence. The maximum mass at $M=M_{\max }^{(\mathrm{B}) \text { stat }}$ (with $P_{\mathrm{c}}=P_{\mathrm{BQ}}$ ) is due is to the strong phase transition, and is somewhat lower than the flat maximum on the $\mathrm{Q}$ branch, $M_{\max }^{(\mathrm{Q}) \text { stat }}$. We proceed to the case of an increasing $J$, when an NS on the $\mathrm{B}$ branch acquires mass and angular momentum from an accretion disk. At some moment it reaches a maximum on the $\mathrm{B}$ branch; it has then $J=J_{1}, M_{\mathrm{b}}^{(\mathrm{B})}=M_{\mathrm{b} 1}$. Under further accretion it collapses into a $\mathrm{Q}$ configuration of the same $M_{\mathrm{b} 1}$ and $J_{1}$, provided $M_{\mathrm{b} 1}<M_{\mathrm{b}, \max }^{(\mathrm{Q})}\left(J_{1}\right)$. The equality is reached exactly at $J_{\text {crit }}$, and for higher $J$ the B star collapses directly into a BH. A critical $J$ is equivalent to maximum $f$, however, which can be reached by the baryon stars. This situation is depicted in detail in Fig. 2 and is discussed further in Sect. 4. A separate question is related to the way in which the NS reaches the instability line. As shown in Zdunik et al. (2005) and Bejger et al. (2011b), the efficiency of transfer of the angular momentum in the process of disk accretion governs the $M(R)$ evolution of a spinning-up NS. To illustrate how an NS can reach the unstable region, we present in Fig. 3 the mass-equatorial radius dependence for evolutionary tracks of accreting NS. A realistic evolutionary path depends on many physical details, such as the configuration and strength of the magnetic field, the accretion rate, and the way the magnetic field interacts with the disk. We assume thin-disk accretion in the presence of the magnetic field by employing a model used previously in Bejger et al. (2011a) and Fortin et al. (2016). Two different efficiencies of angular momentum transfer, $x_{l}=0.5$ and $x_{l}=1$, are considered for initially nonrotating configurations of mass $1.4 M_{\odot}$ and $1.8 M_{\odot}$. The initial magnetic field is $B=10^{8} \mathrm{G}$. If the accretion stops before the instability is reached, the star evolves by moving horizontally from right to left (secular spin-down). Figure 3 is intended to show that it

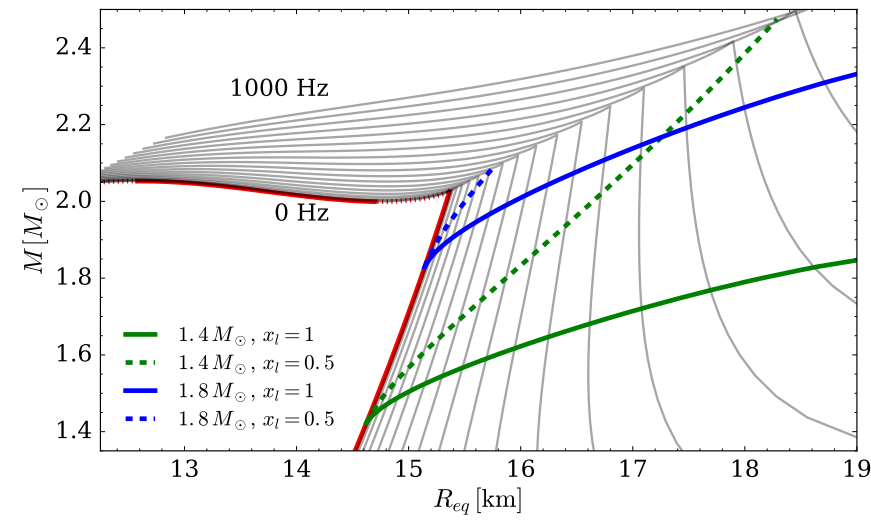

Fig. 3. Evolutionary tracks on the gravitational mass-equatorial radius $M-R_{\text {eq }}$ plane, assuming a thin-disk accretion model, superimposed on the mass-radius curves for constant rotation rates in the range between 0 and $1000 \mathrm{~Hz}$. Two angular momentum transport efficiencies are considered. Solid lines correspond to perfect angular momentum transfer from the accretion disk to the star, dashed lines correspond to half of the accretion-disk angular momentum transferred. The initial masses for these tracks are $1.4 M_{\odot}$ and $1.8 M_{\odot}$. For more details see the text.

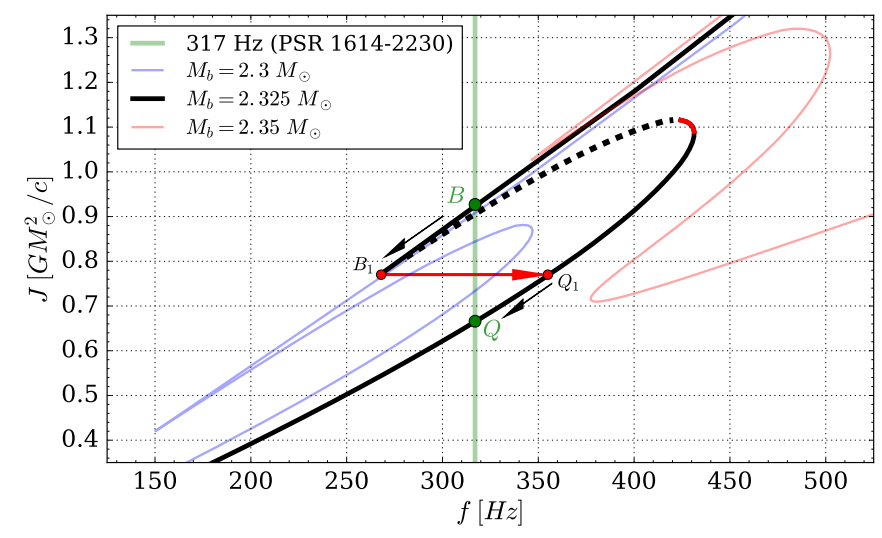

Fig. 4. Evolution of a spinning-down NS with a constant baryon mass $M_{\mathrm{b}}$ on the $J-f$ plane for selected values of $M_{\mathrm{b}}: 2.3 M_{\odot}$ (blue line), $2.325 M_{\odot}$ (black line), and $2.35 M_{\odot}$ (red line). The thick red segment on the $2.325 M_{\odot}$ sequence denotes the back-bending region, which is never reached during the angular momentum loss evolution. Superimposed, the spin frequency of the massive NS PSR 1614-2230. For a detailed description see the text.

is possible to reach the instability region at low or high angular momentum corresponding to the two cases considered in Fig. 2.

The effect of the EOS-induced instability strip may also be studied on the angular momentum-spin frequency $J-f$ plane, assuming a fixed baryon mass sequence, see Fig. 4. The evolution of an isolated NS that decreases its angular momentum $J$ corresponds to a downward movement in this figure. This also means that the central density (and pressure) of such a star increases. A spinning-down NS on the B branch decreases its $J$ until it becomes unstable (point $\mathrm{B}_{1}$ ), which forces it to collapse (dynamically migrate) to another stable branch (point $\mathrm{Q}_{1}$ ) with the same $M_{\mathrm{b}}$ and $J$. We note here that in principle by adopting a certain EOS we may place constraints on the parameters of massive pulsars with known spin frequency. In the example from Fig. 4, an NS with $M_{\mathrm{b}}=2.325 M_{\odot}$ and the spin frequency of PSR 1614-2230 $(317 \mathrm{~Hz})$ is located on either the upper baryonic branch denoted by $\mathrm{B}$, or on the lower branch (hybrid stars $-\mathrm{Q}$ ), the two configurations differing greatly in the 


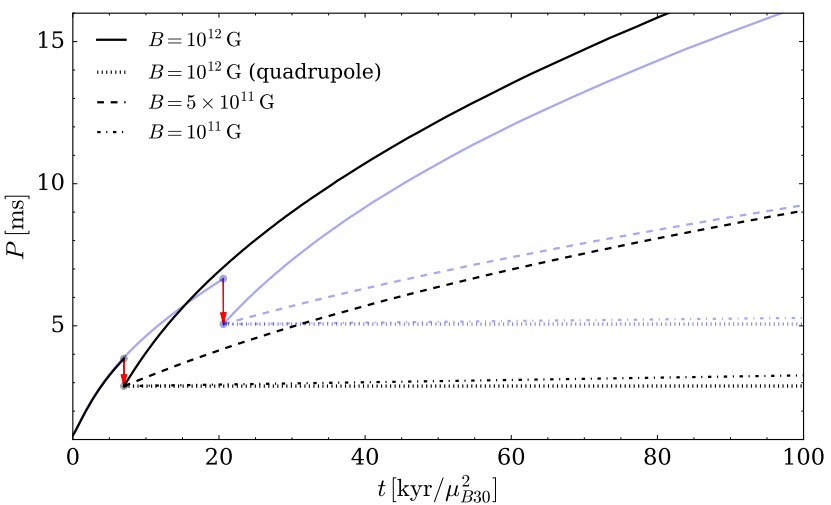

Fig. 5. Evolution of the spin period of a spinning-down NS with constant baryon mass $M_{\mathrm{b}}=2.325 M_{\odot}$ (black lines) and $M_{\mathrm{b}}=2.3 M_{\odot}$ (blue lines), in relation to Fig. 4 . The time unit is normalized by the value of the magnetic dipole moment $\mu_{B}=B R^{3}=10^{30}$ [cgs], which corresponds to a magnetic field of $B=10^{12} \mathrm{G}$ and a stellar radius of $R=10 \mathrm{~km}$. The lines after the mini-collapse related to a sudden spin-up and marked with red arrows are denoted according to the assumption that the $B$ field stayed unchanged (solid lines), decreased twice (to $B=5 \times 10^{11} \mathrm{G}$, dashed lines), or decreased by one order of magnitude (to $B=10^{11} \mathrm{G}$, dash-dotted lines). Dotted lines mark the evolution of a system with a purely quadrupolar magnetic field of $B=10^{12} \mathrm{G}$, emulating a drastic change of the field structure after the mini-collapse.

total angular momentum because of very different moments of inertia. A configuration with a slightly lower $M_{\mathrm{b}}=2.3 M_{\odot}$ (blue line) may, for this particular EOS, exist at $317 \mathrm{~Hz}$ only in the B phase, since the instability transfers it to frequency $\simeq 200 \mathrm{~Hz}$ (significantly lower than $317 \mathrm{~Hz}$ ). A configuration with a slightly higher $M_{\mathrm{b}}=2.35 M_{\odot}$ (red line) is excluded as a model of PSR 1614-2230; during its evolution, it never spins down to reach $317 \mathrm{~Hz}$ : after the dynamical migration and a period of spindown, it will collapse to a $\mathrm{BH}$.

The time evolution of the rotation period of an isolated NS loosing its energy and angular momentum due to the dipole radiation is presented in Fig. 5. The assumption of dipole radiation from the pulsar leads to the formula

$$
\frac{\mathrm{d} E}{\mathrm{~d} t}=-\frac{\mu_{B}^{2} \Omega^{4} \sin ^{2} \alpha}{6 c^{3}}
$$

where $E$ is the energy of rotating pulsar and $\mu_{B}=B R^{3}$ the dipole moment of a star. In the framework of general relativity, we should use the total mass-energy of the star $M c^{2}$ in place of $E$. For the evolution of an isolated NS with a fixed total number of baryons, the relation $\mathrm{d} M=\Omega / c^{2} \mathrm{~d} J$ holds, resulting in the equation for the time evolution,

$\frac{\mathrm{d} \tilde{J}}{\mathrm{~d} \tilde{t}}=-3.34 \times 10^{-9} B_{12}^{2} R_{6}^{6} f_{\mathrm{Hz}}^{3}$,

where $\tilde{J}$ is the stellar angular momentum in the units of $G M_{\odot}^{2} / c$ and $\tilde{t}$ is time in kyr, $B_{12}$ is the magnetic field strength in the units of $10^{12} \mathrm{G}$, and $R_{6}$ is the stellar radius in the units of $10^{6} \mathrm{~cm}$ (the same relation in a Newtonian case could be obtained for $E=\frac{1}{2} I \Omega^{2}$ and $J=I \Omega$, with $I$ being the NS moment of inertia). The timescales of slowing down of a solitary pulsar from milliseconds (close to maximum mass-shedding frequency) down to $\sim 15 \mathrm{~ms}$ before and after the mini-collapse are clearly comparable - we assumed that the magnetic moment $\mu_{B}$ does either not change during the evolution, or that the magnetic field decreases.

A mini-collapse is a dynamical process, provided the $\mathrm{B} \longrightarrow$ Q conversion has detonation character (Haensel et al. 2016). It

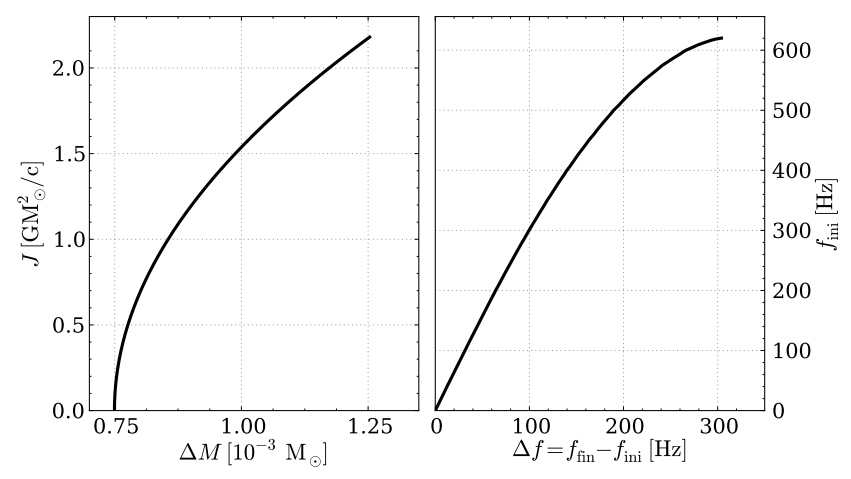

Fig. 6. Left panel: energy release (difference in the gravitational mass) against the angular momentum of the configuration entering the strong phase transition instability. Right panel: spin-up (difference between the final and initial spin frequency) against the spin frequency of the initial configuration. For details see the text.

involves considerable spin-up and a substantial reorganization of the interior of the star (Dimmelmeier et al. 2009). It is also exoenergetic: a substantial amount of energy, quantified as the difference between the initial and the final gravitational mass, $\Delta M=M_{\mathrm{ini}}-M_{\mathrm{fin}}$, is released in the process. The left panel of Fig. 6 shows the relation between the angular momentum $J$ of the star and the energy difference $\Delta M$. The $\Delta M(J)$ relation is approximately quadratic in $J: \Delta M(J)=a J^{2}+\Delta M(0)$. For the particular EOS used in this study, $a=0.106$ and $\Delta M(0)=0.749$, for $\Delta M$ in $10^{-3} M_{\odot}$ and $J$ in $G M_{\odot}^{2} / c$ units. The line ends at a critical $\tilde{J} \approx 2.20$, where the dynamical collapse forces an NS to the instability region, where it collapses to a $\mathrm{BH}$ (see also the right panel of Fig. 2). The right panel of Fig. 6 shows the amount of spin-up (spin frequency difference) $\Delta f=f_{\text {fin }}-f_{\text {ini }}$ that is acquired in the mini-collapse as a function of the initial spin frequency $f_{\text {ini }}$. The situation presented here corresponds to a specific case studied in detail in Zdunik et al. (2008), where the overpressure of the new metastable phase is set to zero, $\delta \bar{P}=0$.

From the same plot one may estimate the "Newtonian" change of kinetic energy and the luminosity of the process. Assuming that the total angular momentum does not change during the process, we obtain

$\Delta E^{\mathrm{rot}}=E_{\mathrm{fin}}^{\mathrm{rot}}-E_{\mathrm{ini}}^{\mathrm{rot}}=\frac{1}{2} J\left(\Omega_{\mathrm{fin}}-\Omega_{\mathrm{ini}}\right)$.

For the value of $J=2 G M_{\odot}^{2} / c$ the change of the spin frequency is approximately $\Delta f=240 \mathrm{~Hz}(\Delta \Omega=1510 \mathrm{rad} / \mathrm{s})$. For these figures we obtain $\Delta E^{\text {rot }} \simeq 2 \times 10^{52} \mathrm{erg}$. This value overestimates the difference in total gravitational mass $\Delta M$ by one order of magnitude $\left(10^{-3} M_{\odot} c^{2} \simeq 2 \times 10^{51} \mathrm{erg}\right)$. The process occurs on a dynamical timescale of a millisecond.

\section{Discussion}

The goal of this article is to bona fide consider a specific class of EOS featuring a substantial phase transition motivated by the theory of dense matter physics. The EOS-induced instability region divides stable NS configurations into two disjoint families (twin families). Its existence has interesting astrophysical consequences for rotating NSs. We note that it facilitates a natural (i.e., not fine-tuned) way for various astrophysical phenomena that we list below.

Spin frequency cutoff. Even though theoretical models of NSs allow for spin rotation rates much above $1 \mathrm{kHz}$ and although 
with current observational techniques such rapidly rotating pulsars could be detected (see, e.g., Patruno 2010; Davoust et al. 2011), so far, the most rapidly rotating NS observed is PSR J1748-2446ad (716 Hz, Hessels et al. 2006). It cannot be excluded a priori that some rapidly rotating and massive NSs were created close to their currently observed state, that is, in a specific type of core-collapse supernovæ. If this were the case, then they might appear practically everywhere on the right side of the thick blue line of Fig. 1, with the exception of the instability strip (red area), where no stationary axisymmetric solutions are possible. However, as the observations, evolutionary arguments, and numerical simulations tend to suggest, NS that become radio pulsars are not born with $\sim 1-3$ ms periods, but with much longer periods of $\sim 20-150 \mathrm{~ms}$, see Faucher-Giguère \& Kaspi (2006), Kramer et al. (2003), Table 7.6 in Lyne \& Graham-Smith (1998), and references therein (a specific class of NS with millisecond periods at birth in massive core-collapse supernovae are thought to be progenitors of magnetars, which are observed as soft-gamma ray repeaters or anomalous X-ray pulsars, see e.g., Kargaltsev \& Pavlov 2008). Then, after slowing down to a period of a few seconds and entering the pulsar graveyard, they gain their angular momentum, as well as mass, during long-term accretion processes in lowmass binary systems (in the so-called recycling of dead pulsars, see Alpar et al. 1982; Radhakrishnan \& Srinivasan 1982; Wijnands \& van der Klis 1998), and it is possible that some of them enter the strong phase transition instability strip sometime in their evolution. Sufficiently massive and sufficiently rapidly rotating NS will then migrate dynamically along the $M_{\mathrm{b}}=$ const. track in the direction of the twin branch (see, e.g., Dimmelmeier et al. 2009). Moreover, for some critical angular momentum (critical spin frequency) the value of $M_{\mathrm{b}}$ on the right side of the instability strip (the $M_{\mathrm{b}}\left(R_{\mathrm{eq}}\right)$ peaks in Fig. 1) is higher than the corresponding maximum of $M_{\mathrm{b}}$ on the twin branch - in that case, the star collapses to a $\mathrm{BH}$.

No observations of back-bending in radio-pulsar timing. One of the observational predictions related to substantial densematter phase transitions is the detection of the back-bending phenomenon, which occurs at spin frequencies of known pulsars. As we showed in Sect. 3, Fig. 1, NSs exhibiting an instability that is caused by a strong phase transition avoid the vast majority of the back-bending region for spin frequencies lower than some critical value. The most rapidly rotating currently known pulsar, PSR J1748-2446ad, has a spin period of $716 \mathrm{~Hz}$. From Fig. 1 we note that the $f=700 \mathrm{~Hz}$ line is the first dashed line above the no back-bending (wavy pattern) region. When we assume that the EOS used for illustration is the true EOS of dense matter, this means that PSR J1748-2446ad, which does not show the features of back-bending in the timing, still resides on the hadronic branch (does not contain the quark core). Additionally, the most massive stars that are in the back-bending region may not be effective pulsars - they may be electromagnetically exhausted, with their magnetic field dissipated in the violent process of mini-collapse, and therefore not easily detectable.

Radius gap. The existence of an instability strip creates in a mass- and spin-frequency-dependent radius region of avoidance between the allowed green region and stable baryonic branch on the right of Fig. 1, which is broadened with increasing mass (see also Fig. 3 of Benic et al. 2015). For nonrotating $2 M_{\odot}$ NS, the predicted $R$-gap is $\sim 1 \mathrm{~km}$. Small-radius Q-branch twins have $R^{(\mathrm{Q})}=12.5-14.5 \mathrm{~km}$ within a very narrow mass range $\sim 0.1 M_{\odot}$. The measurement of a radius $R>15 \mathrm{~km}$ for a $\sim 2 M_{\odot}$ star indicates a B-branch configuration. If for another $\sim 2 M_{\odot}$ NS the

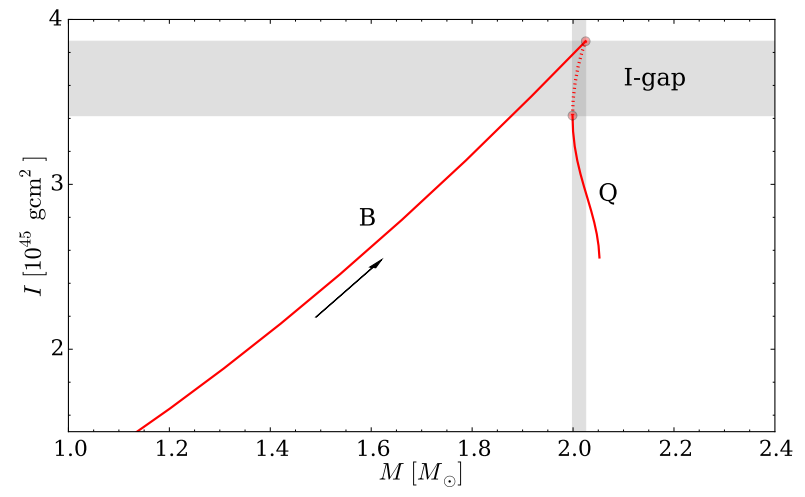

Fig. 7. Stellar moment inertia $I$ versus gravitational mass $M$ for nonrotating NS models. Solid lines: stable equilibrium configurations. The arrow indicates increasing central density. The upper filled circle corresponds to the termination of the stable B branch, and the lower circle correspond to the first stable equilibrium configuration of the $\mathrm{Q}$ branch. The Q-branch line ends at the last stable equilibrium configuration. The dotted line corresponds to unstable configurations. The $I$ - gap between the stable B and Q twin branches is shown. For details see the text.

radius is determined to be within the range $12.5-14.5 \mathrm{~km}$, then we obtain strong evidence in favor of distinct $\mathrm{B}$ and $\mathrm{Q}$ twin branches as a result of a strong $\mathrm{B} \longrightarrow \mathrm{Q}$ phase transition.

Moment of inertia gap. The twins on the B and $\mathrm{Q}$ branches have different internal structure. The B-star twin is more compact, and its mass is concentrated in the dense quark core. Consequently, at the same $M$ of twins, one has $I^{(\mathrm{Q})}$ significantly smaller than $I^{(\mathrm{B})}$, as shown in Fig. 7. Moreover, the strong first-order phase transition B $\longrightarrow$ Q results in an I gap between the B and Q twins (Fig. 7). The moment of inertia of NS can be measured through the spin-orbit effect contribution to the timing parameters for a binary of two radio pulsars (Damour \& Schaefer 1988). The first binary of this type, PSR J0737-3039A,B was discovered more than a decade ago (Lyne et al. 2004). Pulsar B has become invisible to terrestrial observers in March 2008 because its beam wandered out of our line of sight as a consequence of the geodetic precession effect (Perera et al. 2010). It may reappear as late as in 2034 (or later), depending on the model of the pulsar magnetosphere (see, e.g., Lomiashvili \& Lyutikov 2014). Since the mass of pulsar A has been accurately determined, a measurement of its moment of inertia through the spin-orbit momentum coupling would allow us to constrain the radius and hence the EOS (Lattimer \& Schutz 2005). Given the present timing accuracy of the system's post-Keplerian parameters, that is, the periastron advance, the decrease in the orbital period and the Shapiro shape parameter, from which the spin-orbit coupling contribution is derived, reasonable accuracy may be achieved around the time of pulsar B reappearance in $\simeq 20 \mathrm{yr}$ (Kramer \& Wex 2009). However, in the forthcoming era of large radio telescopes (e.g., FAST, SKA) the number of known pulsars will increase by orders of magnitude, including many thousands of millisecond pulsars, out of which we may hopefully expect tens of binary systems with two pulsars suitable for simultaneously measuring $I$ and $M$ of an NS. A sufficiently dense set of pairs $\left\{I_{i}, M_{i}\right\}$ resulting from these future measurements could then be used to confirm or reject the generic $I(M)$ shape in Fig. 7.

EOS-induced dynamical collapse as an energy reservoir. We assume that the $\mathrm{B} \longrightarrow \mathrm{Q}$ phase transition, mediated by strong interaction, occurs in the detonation regime. A dynamical process involving NS, triggered by the loss of stability, is associated with a substantial energy release $\left(\sim 10^{52} \mathrm{erg}\right)$, heating of 
dense matter, kinetic energy flow, and some emission of radiation, on both a short time-scale (NS quake, mini-collapse) and long time-scale (surface glowing). The process is probably also associated with a substantial rearrangement of the NS magnetic field. For NS with total angular momenta larger than some critical value, it leads to a direct collapse to a $\mathrm{BH}$. This event is related to the expulsion of the magnetic field and thus the dynamical migration to the high-mass twin branch may be considered a natural extension of the Falcke \& Rezzolla (2014) cataclysmic scenario. Alternatively, for NSs below the angular-momentum threshold, the mini-collapse dynamics may influence the magnetic field to such an extent that it becomes a transient source of observable magnetospheric emission after the final configuration ends up on the twin branch.

To conclude, the assumption of the strong phase transition in the NS EOS leads to a number of falsifiable (at least in principle) astrophysical predictions. As we described in Sect. 3 and in Fig. 4, a transition to a new exotic phase (deconfined quarks in this example) constrains the range of available $M_{\mathrm{b}}$ and $J$. This reasoning can be extended to other EOS functionals, like the moment of inertia $I$; moreover, these constraints may be combined with the observations that are sensitive to the composition of the core, for example, NS cooling studies. The evolutionary scenario in which some of NS collapse to BHs produces a specific NS-BH mass function without a mass gap, which should be possible to test with current and future searches for low-mass BHs with microlensing surveys (Wyrzykowski et al. 2016), for instance. Unless the NS magnetic field is amplified and/or reoriented during the mini-collapse event, it is likely that it is dissipated and disordered during the process - in the latter case, we expect a population of massive ineffective pulsars with a low magnetic field. Moreover, a dynamical mini-collapse creates a characteristic signature of the GW emission, strongly dependent on the EOS and mass of the NS; short transient GW radiation of this type should be detectable by the advanced era interferometric detectors (Dimmelmeier et al. 2009). If the collapse is not entirely axisymmetric, the final configuration may retain an asymmetry, thus creating a rotating NS that spins down while continuously emitting the almost-monochromatic GWs. Such objects are among the prime astrophysical targets of the Advanced LIGO and Advanced Virgo interferometric detectors (see the demonstration of search pipelines used on the initial LIGO and initial Virgo detector data, e.g., Aasi et al. 2014a,b, 2015).

There are several open questions related to the various aspects of the high-mass twin scenario, such as what the conditions are for the NS to reach the instability region via the disk accretion spin-up, what the influence of the possible meta-stability of the quark phase core is on the NS population on the twin branch, and how the electromagnetic and gravitational-wave emission depends on the parameters of the NS. These questions will be addressed in subsequent studies.

Acknowledgements. This work has been supported in part by the Polish Nationa Science Centre (NCN) under grant No. UMO-2014/13/B/ST9/02621. D.B. has been supported in part by the MEPhI Academic Excellence Project under contract No. 02.a03.21.0005. The authors acknowledge support from the COST Action MP1304 "NewCompStar" for their networking activities.

\section{References}

Aasi, J., Abbott, B. P., Abbott, R., et al. 2014a, Class. Quantum Gray., 31, 165014 Aasi, J., Abbott, B. P., Abbott, R., et al. 2014b, Phys. Rev. D, 90, 062010

Aasi, J., Abbott, B. P., Abbott, R., et al. 2015, ApJ, 813, 39

Abuki, H., Baym, G., Hatsuda, T., \& Yamamoto, N. 2010, Phys. Rev. D, 81, 125010
Alpar, M. A., Cheng, A. F., Ruderman, M. A., \& Shaham, J. 1982, Nature, 300, 728

Alford, M., Han, S., \& Prakash, M. 2013, Phys. Rev. D, 88, 083013

Alvarez-Castillo, D. E., \& Blaschke, D. 2013, ArXiv e-prints [arXiv: 1304.7758]

Alvarez-Castillo, D. E., \& Blaschke, D. 2015, Phys. Part. Nucl. 46, 846

Alvarez-Castillo D. E., Ayriyan A., Benic S., et al. 2016, Eur. Phys. J. A, 52, 69

Alvarez-Castillo, D., Benic, S., Blaschke, D., Han, S., \& Typel, S. 2016, Eur. Phys. J. A, 52, 232

Andronic, A., Blaschke, D., Braun-Munzinger, P., et al. 2010, Nucl. Phys. A, 837,65

Antoniadis, J., Freire, P. C. C., Wex, N., et al. 2013, Science, 340, 448

Arzoumanian, Z., Gendreau, K. C., Baker, C. L., et al. 2014, Space Telescopes and Instrumentation 2014: Ultraviolet to Gamma Ray, Proc. SPIE, 9144, 914420

Batyuk, P., Blaschke, D., Bleicher, M., et al. 2016, Phys. Rev. C, 94, 4917

Bauswein, A., Stergioulas, N., \& Janka, H.-T. 2015, arXiv e-prints [arXiv: 1503.08769]

Bazavov, A., Bhattacharya, T., DeTar, C., et al. (HotQCD Collaboration) 2014, Phys. Rev. D, 90, 4503

Bejger, M., Gondek-Rosińska, D., Gourgoulhon, E., et al. 2005, A\&A, 431, 297 Bejger, M., Fortin, M., Haensel, P., \& Zdunik, J. L. 2011a, A\&A, 536, A87 Bejger, M., Zdunik, J. L., Haensel, P., \& Fortin, M. 2011b, A\&A, 536, A92 Benic, S. 2014, Eur. Phys. J. A, 50, 111

Benic, S., Blaschke, D., Alvarez-Castillo, D. E., Fischer, T., \& Typel, S. 2015, A\&A, 577, A40

Blaschke, D., Alvarez-Castillo, D. E., \& Benic, S. 2013, arXiv e-prints [arXiv: 1310.3803 ]

Bogdanov S. 2013, ApJ, 762, 96

Bonazzola, S., \& Gourgoulhon, E. 1994, Class. Quantum Gray., 11, 1775

Bonazzola, S., Gourgoulhon, E., Salgado, M., \& Marck, J. A. 1993, A\&A, 278, 421

Borsanyi, S., Fodor, Z., Hoelbling, C., et al. 2014, Phys. Lett. B, 730, 99

Chubarian, E., Grigorian, H., Poghosyan, G. S. \& Blaschke, D. 2000, A\&A, 357, 968

Damour, T., \& Schaefer, G. 1988, Nuovo Cimento B, 101, 127

Damour, T., Nagar, A., \& Villain, L. 2012, Phys. Rev. D, 85, 123007

Davoust, E., Petit, G., \& Fayard, T. 2011, A\&A, 534, A7

Demorest P. B., Pennucci, T., Ransom, S. M., Roberts, M. S. E., \& Hessels, J. W. T. 2010, Nature, 467, 1081

Dimmelmeier, H., Bejger, M., Haensel, P., \& Zdunik, J. L. 2009, MNRAS, 396, 2269

Elshamouty, K., Heinke, C., Morsink, S., Bogdanov, S., \& Stevens, A. 2016, ApJ, 826, 162

Endo, T., Maruyama, T., Chiba, S., \& Tatsumi, T. 2006, Prog. Theor. Phys., 115, 337

Falcke, H., \& Rezzolla, L. 2014, A\&A, 562, A137

Faucher-Giguère, C.-A., \& Kaspi, V. M. 2006, ApJ, 643, 332

Feroci, M., den Herder, J. W., Bozzo, E., et al. 2012, Proc. SPIE, 8443, 84432

Fonseca, E., Pennucci, T. T., Ellis, J. A., et al. 2016, ApJ, 832, 167

Fortin, M., Zdunik, J. L., Haensel, P., \& Bejger, M. 2015, A\&A, 576, A68

Fortin, M., Zdunik, J. L., Haensel, P., \& Bejger, M. 2016a, A\&A, 586, A109

Fortin, M., Providencia, C., Raduta, A. R., et al. 2016b, Phys. Rev. C, 94, 5804

Friedman, J. L., Ipser, J. R., \& Sorkin, R. D., 1988, ApJ, 325, 722

Friman, B., Höhne, C., Knoll, J., et al. 2011, Lect. Notes Phys., 814, 1

Gazdzicki, M., et al. [NA49-future Collaboration] 2006, ArXiv e-prints [arXiv:nucl-ex/0612007]

Glendenning, N. K., Pei, S., \& Weber, F. 1997, Phys. Rev. Lett., 79, 1603

Guillot, S., Servillat, M., Webb, N. A., \& Rutledge, R. E. 2013, ApJ, 772, 7

Hatsuda, T., Tachibana, M., Yamamoto, N., \& Baym, G. 2006, Phys. Rev. Lett., 97, 122001

Haensel, P., Potekhin, A. Y., \& Yakovlev, D. G. 2007, Neutron Stars 1: Equation of State and Structure (New York: Springer)

Haensel, P., Bejger, M., Fortin, M., \& Zdunik, J. L. 2016, Eur. Phys. J. A, 52, 59

Heinke, C. O., Cohn, H. N., Lugger, P. M., et al. 2014, MNRAS, 444, 443

Hessels, J. W. T., Ransom, S. M., Stairs, I. H., et al. 2006, Science, 311, 1901

Ivanov, Y. B., 2013, Phys. Lett. B, 721, 123

Ivanov, Y. B., \& Blaschke, D. 2015, Phys. Rev. C, 92, 024916

Kargaltsev, O., \& Pavlov, G. G., 2008, 40 Years of Pulsars: Millisecond Pulsars, Magnetars and More, AIP Conf. Proc., 983, 171

Klähn, T., Blaschke, D., Typel, S., et al. 2006, Phys. Rev. C, 74, 035802

Klähn, T., Blaschke, D., \& Weber, F. 2012, Phys. Part. Nucl. Lett., 9, 484

Kojo, T., 2016, Eur. Phys. J. A, 52, 51

Kramer, M., \& Wex, N. 2009, Class. Quantum Gray., 26, 073001

Kramer, M., Lyne, A. G., Hobbs, G., et al. 2003, ApJ, 593, L31

Lattimer, J. M., \& Schutz, B. 2005, ApJ, 629, 979

Lindblom, L. 1998, Phys. Rev. D, 58, 024008

Lomiashvili, D., \& Lyutikov, M. 2014, MNRAS, 441, 690 
M. Bejger et al.: Consequences of phase transitions in rotating NSs

Lyne, A. G., \& Graham-Smith, F. 1998, Pulsar astronomy (Cambridge University Press)

Lyne, A. G., Burgay, M., Kramer, M., et al. 2004, Science, 303, 1153

Masuda, K., Hatsuda, T. \& Takatsuka, T. 2013, PTEP, 2013, 073 D01

McLerran, L. \& Pisarski, R. D. 2007, Nucl. Phys. A, 796, 83

Miller, M. C. \& Lamb, F. K. 2016, Eur. Phys. J. A, 52, 63

Motch, C., Wilms, J., Barret, D., et al. 2013, ArXiv e-prints [arXiv: 1306.2334]

Oppenheimer, J. R., \& Volkoff, G. M. 1939, Phys. Rev., 55, 374

Patruno, A. 2010, in Proc. High Time Resolution Astrophysics, The Era of Extremely Large Telescopes (HTRA-IV), May 5-7, Agios Nikolaos, Crete Greece, 28

Perera, B. B. P., McLaughlin, M. A., Kramer, M., et al. 2010, ApJ, 721, 1193

Radhakrishnan V., \& Srinivasan G. 1982, Curr. Sci., 51, 1096

Röpke, G., Blaschke, D. \& Schulz, H. 1986, Phys. Rev. D, 34, 3499

Schäfer, T., \& Wilczek, F. 1999, Phys. Rev. Lett., 82, 3956

Schulz, H., \& Röpke, G. 1987, Z. Phys. C, 35, 379

Seidov, Z. F. 1971, Sov. Astron. Lett., 15, 347

Sissakian, A. N., Sorin, A. S., \& Toneev, V. D. 2006, Conf. Proc. C, 060726, 421
Sorkin, R. 1981, ApJ, 249, 254

Sorkin, R. D. 1982, ApJ, 257, 847

Stephans, G. S. F. 2006, J. Phys. G, 32, S447

Tolman, R. C. 1939, Phys. Rev., 55, 364

Typel, S., Röpke, G., Klähn, T., Blaschke, D., \& Wolter, H. H. 2010, Phys. Rev. C, 81, 015803

Watts, A., Espinoza, C. M., Xu, R., et al. 2015, Proc. Advancing Astrophysics with the Square Kilometre Array (AASKA14), 9-13 June, Giardini Naxos, Italy, 043

Wijnands, R., \& van der Klis, M. 1998, Nature, 394, 344

Wyrzykowski, Ł., Kostrzewa-Rutkowska, Z., Skowron, J., et al. 2016, MNRAS, 458, 3012

Yamamoto, Y., Furumoto, T., Yasutake, N. \& Rijken, T. A. 2016, Eur. Phys. J. A, 52, 19

Zdunik, J. L., Haensel, P., Gourgoulhon, E., \& Bejger, M. 2004, A\&A, 416, 1013 Zdunik, J. L., Haensel, P., \& Bejger, M. 2005, A\&A, 441, 207

Zdunik, J. L., Bejger, M., Haensel, P., \& Gourgoulhon, E. 2006, A\&A, 450, 747

Zdunik, J. L., Bejger, M., Haensel, P., \& Gourgoulhon, E. 2008, A\&A, 479, 515 